\title{
Combined electroweak and QCD fit to HERA data
}

\author{
I. Abt, ${ }^{1}$ A. M. Cooper-Sarkar, ${ }^{2}$ B. Foster, ${ }^{2,3,4}$ C. Gwenlan, ${ }^{2}$ V. Myronenko, ${ }^{4}$ O. Turkot, ${ }^{4}$ and K. Wichmann ${ }^{4}$ \\ ${ }^{1}$ Max-Planck-Institut für Physik, Werner-Heisenberg-Institut, 80805 München, Germany \\ ${ }^{2}$ Physics Department, University of Oxford, Oxford, OX1 3RH United Kingdom \\ ${ }^{3}$ Hamburg University, I. Institute of Experimental Physics, 22671 Hamburg, Germany \\ ${ }^{4}$ Deutsches Elektronen Synchrotron DESY, 22671 Hamburg, Germany
}

(Received 19 April 2016; published 15 September 2016)

\begin{abstract}
A simultaneous electroweak and QCD fit of electroweak parameters and parton distribution functions to HERA data on deep inelastic scattering is presented. The input data are neutral current and charged current inclusive cross sections measured by the H1 and ZEUS collaborations at the ep collider HERA. The polarization of the electron beam was taken into account for the ZEUS and H1 data recorded between 2004 and 2007. Results are presented on the vector and axial-vector couplings of the $Z$ boson to $u$ - and $d$-type quarks. The values are in agreement with Standard Model predictions. The results on $a_{u}$ and $v_{u}$ represent the most precise measurements from a single process.
\end{abstract}

DOI: 10.1103/PhysRevD.94.052007

\section{INTRODUCTION}

Data on deep inelastic electron ${ }^{1}$-proton, $e p$, scattering (DIS) have been used in analyses within the framework of quantum chromo dynamics (QCD) for many years [1] and have formed the basis of investigations of the structure of the proton. The data from the ep collider HERA extended the reach in the four-momentum-transfer squared, $Q^{2}$, and in Bjorken $x, x_{B j}$, by several orders of magnitude with respect to previous fixed-target experiments [2]. At HERA, values of $Q^{2}$ of up to $50000 \mathrm{GeV}^{2}$ were reached, a regime where the contribution of $Z$ exchange becomes comparable to the contribution from photon exchange.

During the HERA II running period, the HERA collider provided a significant amount of data with beams longitudinally polarized to an average level between 25\% and $35 \%$. This facilitates detailed studies of electroweak (EW) effects. Recently, the ZEUS collaboration published a combined QCD and electroweak analysis [3] exploiting the ZEUS neutral current (NC) and charged current (CC) $e^{+} p$ and $e^{-} p$ inclusive cross sections for polarized beams [4-7]. For the analysis presented here, cross sections published by the $\mathrm{H} 1$ collaboration [8] for polarized beams were also considered.

A combined QCD and EW fit to the H1 and ZEUS data was performed in which PDF parameters and EW parameters, specifically the couplings of the $Z$ boson to $u$ - and $d$-type quarks, were varied simultaneously, such that correlations between PDF and electroweak parameters were properly treated. In the present paper, the use of $\mathrm{H} 1$ and ZEUS polarized data is expected to improve the precision of the vector couplings of the $Z$ boson as detailed in Sec. II.

\footnotetext{
${ }^{1}$ In this paper, the word "electron" refers to both electrons and positrons, unless otherwise stated.
}

\section{QCD AND EW COMBINED ANALYSIS}

The analysis presented here follows closely the method described in detail in the ZEUS publication [3]. It uses the next-to-leading-order (NLO) DGLAP [9-13] formalism to describe the evolution of the parton distribution functions (PDFs) with $Q^{2}$ and the on-shell definition of $\sin ^{2} \theta_{W}=1-M_{W}^{2} / M_{Z}^{2}$, where $\sin ^{2} \theta_{W}$ is the electroweak mixing angle and $M_{W}$ and $M_{Z}$ are the mass of the $W$ and $Z$ boson, respectively. The EW part of the analysis was performed at leading order with partial higher-order corrections in the on-shell scheme as calculated by EPRC [14]. The RT variable-number heavy-flavor scheme [15-17] was employed and the values of PDG14 [18] were used for all masses and couplings throughout the analysis, unless they were free parameters in a fit.

Following the ZEUS QCD and EW analysis [3], the PDFs of the proton were parametrized with 13 free parameters as

$$
\begin{gathered}
x g(x)=A_{g} x^{B_{g}}(1-x)^{C_{g}}-A_{g}^{\prime} x^{B_{g}^{\prime}}(1-x)^{C_{g}^{\prime}}, \\
x u_{v}(x)=A_{u_{v}} x^{B_{u_{v}}}(1-x)^{C_{u_{v}}}\left(1+E_{u_{v}} x^{2}\right), \\
x d_{v}(x)=A_{d_{v}} x^{B_{d_{v}}}(1-x)^{C_{d_{v}}}, \\
x \bar{U}(x)=A_{\bar{U}} x^{B}(1-x)^{C_{\bar{U}}}, \\
x \bar{D}(x)=A_{\bar{D}} x^{B}(1-x)^{C_{\bar{D}}},
\end{gathered}
$$

where $x$ is the fraction of the proton momentum carried by the quark. The normalization parameters, $A_{u_{v}}, A_{d_{v}}, A_{g}$, are constrained by the quark-number sum rules and the momentum sum rule. The strange-quark distribution is expressed as an $x$-independent fraction, $f_{s}$, of the $d$-type 
sea, $x \bar{s}=0.4 x \bar{D}$ at the starting scale $\mu_{\mathrm{f}_{0}}^{2}=1.9 \mathrm{GeV}^{2}$. The parameter $C_{g}^{\prime}$ is fixed to $C_{g}^{\prime}=25$ [19].

The PDF parameters and the axial-vector and vector couplings of the $Z$ boson to the $u$ - and $d$-type quarks, $a_{u}$, $a_{d}, v_{u}$ and $v_{d}$, were fitted to the HERA inclusive crosssection data. For this fit, called HH-EW-Z, the ZEUSfitter package $^{2}$ was used. The results were cross-checked with the HERAFitter [20] package.

In the HH-EW-Z fit, the CC data add to determination of the PDF parameters whereas the NC data serve to determine both the PDF parameters and the EW couplings. The NC data were published as reduced cross sections which were defined for $e^{-} p$ and $e^{+} p$ scattering in terms of the generalized structure functions $\tilde{F}_{2}, x \tilde{F}_{3}, F_{L}$, as

$$
\begin{aligned}
\sigma_{r, \mathrm{NC}}^{e^{ \pm} p}= & \frac{x_{\mathrm{Bj}} Q^{4}}{2 \pi \alpha^{2}} \frac{1}{Y_{+}} \frac{d^{2} \sigma\left(e^{ \pm} p\right)}{d x_{\mathrm{Bj}} d Q^{2}} \\
= & \tilde{F}_{2}\left(x_{\mathrm{Bj}}, Q^{2}\right) \mp \frac{Y_{-}}{Y_{+}} x \tilde{F}_{3}\left(x_{\mathrm{Bj}}, Q^{2}\right) \\
& -\frac{y^{2}}{Y_{+}} F_{L}\left(x_{\mathrm{Bj}}, Q^{2}\right)
\end{aligned}
$$

where $x_{\mathrm{Bj}}$ is the Bjorken scaling variable and $y=P \cdot q / P$. $k$ is the fraction of the electron energy transferred to the proton in the rest frame of the proton. At HERA energies, the mass of the incoming electrons (protons) with energies $E_{e}\left(E_{p}\right)$ can be neglected and the variables $Q^{2}, x_{\mathrm{Bj}}$ and $y$ are related as $Q^{2}=s x_{\mathrm{Bj}} y$, where $s=4 E_{e} E_{p}$ is the square of the electron-proton center-of-mass energy.

The generalized structure functions depend on the longitudinal polarization of the electron beam, which is defined as

$$
P_{e}=\frac{N_{R}-N_{L}}{N_{R}+N_{L}}
$$

where $N_{R}$ and $N_{L}$ are the numbers of right- and left-handed electrons in the beam. It is useful to split the contributions to the structure functions into unpolarized and polarized pieces, $\tilde{F}_{2}=\tilde{F}_{2}{ }^{0}+P_{e} \tilde{F}_{2}{ }^{P}$ and $x \tilde{F}_{3}=x \tilde{F}_{3}{ }^{0}+P_{e} x \tilde{F}_{3}{ }^{P}$, where, at leading order in $\mathrm{QCD},{ }^{3}$ the unpolarized pieces are given by

$$
\begin{gathered}
\tilde{F}_{2}{ }^{0}=\sum_{i} A_{i}^{0}\left(Q^{2}\right)\left[x q_{i}\left(z, Q^{2}\right)+x \bar{q}_{i}\left(x, Q^{2}\right)\right], \\
x \tilde{F}_{3}^{0}=\sum_{i} B_{i}^{0}\left(Q^{2}\right)\left[x q_{i}\left(z, Q^{2}\right)-x \bar{q}_{i}\left(x, Q^{2}\right)\right],
\end{gathered}
$$

\footnotetext{
${ }^{2}$ The package was also used in the combined ZEUS electroweak and QCD analysis [3].

${ }^{3}$ The QCD analysis is performed at NLO, but the LO expressions illustrate the dominant dependencies on the couplings.
}
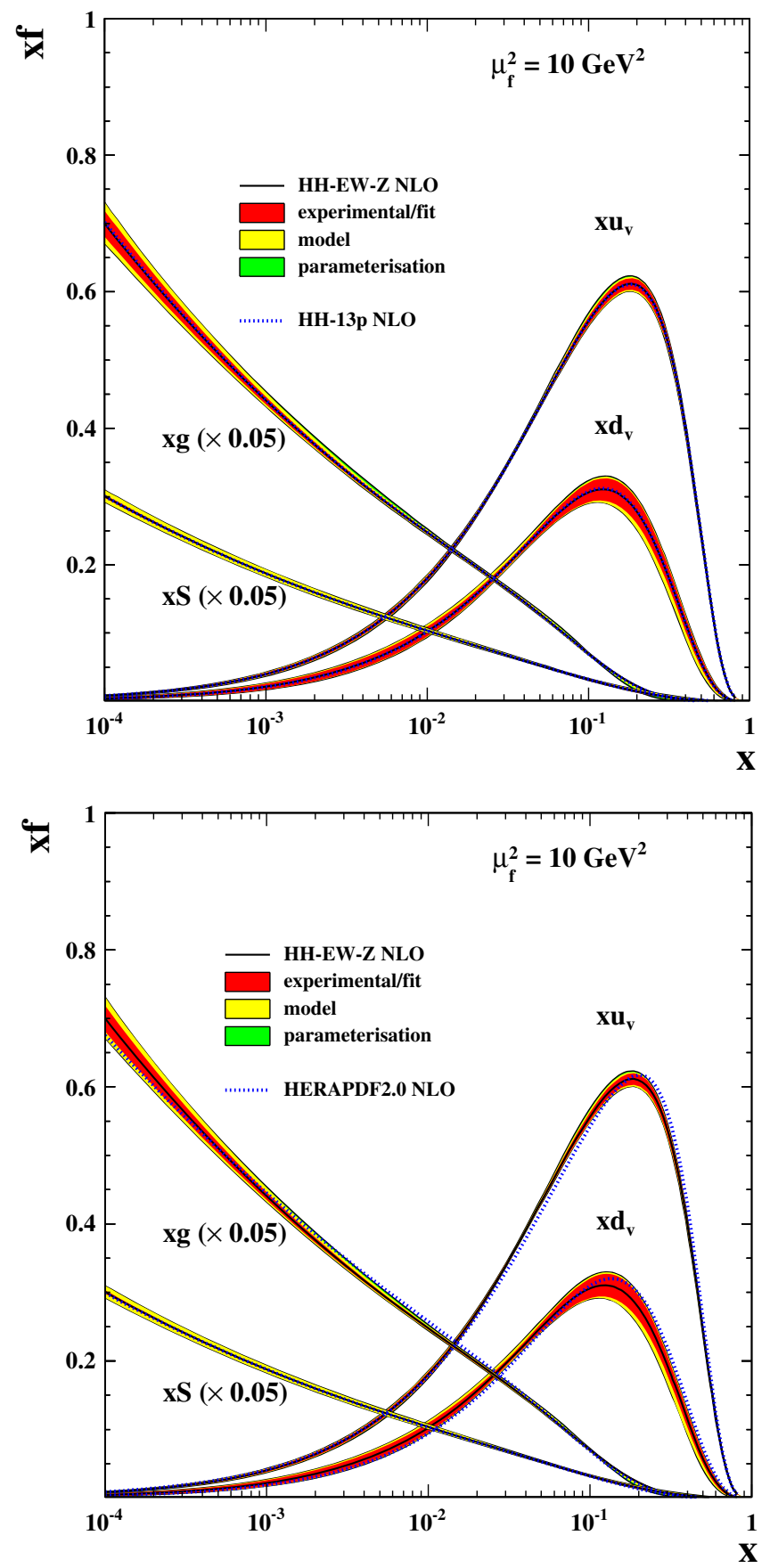

FIG. 1. The NLO PDF set HH-EW-Z with cumulative experimental/fit, model and parametrization uncertainties at the factorization scale $\mu_{\mathrm{f}}^{2}=10 \mathrm{GeV}^{2}$. All positive and negative uncertainties in the model were added separately in quadrature. The parametrization uncertainty represents an envelope of all individual parametrization uncertainties. Also shown are the central values of HH-13p (top) and HERAPDF2.0 NLO (bottom).

where the sum runs over $u$ - and $d$-type quarks and

$A_{i}^{0}\left(Q^{2}\right)=e_{i}^{2}-2 e_{i} v_{i} v_{e} \chi_{Z}+\left(v_{e}^{2}+a_{e}^{2}\right)\left(v_{i}^{2}+a_{i}^{2}\right) \chi_{Z}^{2}$, 
TABLE I. The correlation matrix of all parameters of the HH-EW-Z fit.

\begin{tabular}{|c|c|c|c|c|c|c|c|c|c|c|c|c|c|c|c|c|c|}
\hline Para & $x g: B$ & $g: C$ & $x g: A^{\prime}$ & $x g: B^{\prime}$ & $x u_{v}: 1$ & $u_{v}: C$ & $u_{v}$ & $d_{v}: B$ & $l_{v}:$ & U: $C$ & $x \bar{D}: A$ & $x \bar{D}: B$ & $x \bar{D}: C$ & $a_{u}$ & $a_{d}$ & $v_{u}$ & $v_{d}$ \\
\hline$B$ & 1.000 & 491 & .224 & 0.935 & 012 & 0.106 & 0.044 & 0.049 & .078 & 0.049 & -0.098 & -0.140 & 0.018 & 0.057 & 0.061 & 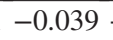 & -0.0 \\
\hline$C$ & 0.491 & .000 & 0.660 & 0.707 & .287 & .267 & -0.464 & 0.054 & 0.196 & -0.047 & -0.140 & 0.175 & -0.369 & 0.106 & 0.093 & 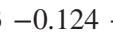 & -0.114 \\
\hline$A^{\prime}$ & -0.224 & 0.660 & 1.000 & 0.125 & .513 & .361 & -0.593 & 0.226 & 0.254 & 0.162 & 0.084 & 0.072 & -0.100 & -0.038 & 0.003 & -0 & -0.070 \\
\hline$: B^{\prime}$ & 0.935 & 0.707 & 0.125 & 1.000 & .200 & -0.002 & -0.144 & 0.048 & -0.008 & 0.042 & -0.017 & -0.056 & 0.018 & 0.033 & 0.057 & -0 & -0.074 \\
\hline$u_{v}: B$ & 0.012 & 0.287 & 0.513 & 0.200 & 1.000 & -0.337 & -0.760 & 0.510 & -0.084 & 0.698 & 0.498 & 0.409 & 0.507 & -0.256 & -0.095 & 0.019 & -0.032 \\
\hline$x u_{v}: C$ & 0.106 & -0.267 & -0.361 & -0.002 & -0.337 & 1.000 & 0.796 & -0.249 & -0.247 & -0.140 & 55 & 32 & -0.013 & 0.092 & 0.044 & 0.026 & 0.013 \\
\hline$u_{v}: E$ & 0.044 & -0.464 & -0.593 & -0.144 & -0.760 & 0.796 & 1.000 & -0.298 & -0.057 & -0 . & 65 & 05 & -0.127 & 0.133 & 0.045 & 0.024 & 0.043 \\
\hline$d_{v}: B$ & -0.049 & -0.054 & 0.226 & 0.048 & 0.510 & -0.249 & -0.298 & 1.000 & 0.502 & 0.437 & 0.406 & 0.344 & 0.727 & -0.221 & -0.056 & 0.014 & -0.056 \\
\hline$d_{v}: C$ & -0.078 & 0.196 & 0.254 & -0.008 & -0.084 & -0.247 & -0.057 & 0.502 & 1.000 & -0.116 & -0.168 & -0.175 & -0.097 & 0.107 & 0.115 & -0.092 & -0.109 \\
\hline$I: C$ & -0.049 & -0.047 & 0.162 & 0.042 & 698 & -0.140 & -0.363 & 0.437 & -0.116 & 1.000 & 0.685 & 0.647 & 0.366 & -0.234 & -0 . & -0 & -0.028 \\
\hline $\bar{D}: A$ & -0.098 & -0.140 & 0.084 & -0.017 & 98 & - & -0 & 0.406 & -0 . & 0.685 & 1.000 & 0.961 & 0.525 & & & 49 & 0.021 \\
\hline$x \bar{D}: B$ & -0.140 & -0.175 & 0.072 & -0.056 & 0.409 & -0.032 & -0.105 & 0.344 & -0.175 & 0.647 & 0.961 & 1.000 & 0.460 & -0 & -0.106 & 0.046 & 0.026 \\
\hline$x \bar{D}: C$ & 0.018 & -0.369 & -0.100 & 0.018 & 0.507 & -0.013 & -0.127 & 0.727 & -0.097 & 0.366 & 0.525 & 0.460 & 1.000 & -0.327 & -0.168 & 0.133 & 0.056 \\
\hline$a_{u}$ & 0.057 & 0.106 & -0.038 & 0.033 & -0.256 & 0.092 & 0.133 & -0.221 & 0.107 & -0.234 & -0.231 & -0.210 & -0.327 & 1.000 & 0.928 & -0.665 & -0.779 \\
\hline$a_{d}$ & 0.061 & 0.093 & 0.003 & 0.057 & -0.095 & 0.044 & 0.045 & -0.056 & 0.115 & -0.082 & -0.114 & -0.106 & -0.168 & 0.928 & 1.000 & -0 & -0.876 \\
\hline$v_{u}$ & -0.039 & -0.124 & -0.065 & -0.058 & 0.019 & 0.026 & 0.024 & 0.014 & -0.092 & -0.006 & 0.049 & 0.046 & 0.133 & -0.665 & -0.714 & 0 & 0.880 \\
\hline$v_{d}$ & -0.051 & -0.114 & -0.070 & -0.074 & -0.032 & 0.013 & 0.043 & -0.056 & -0.109 & -0.028 & 0.021 & 0.026 & 0.056 & -0.779 & -0.876 & 0.880 & 1.000 \\
\hline
\end{tabular}

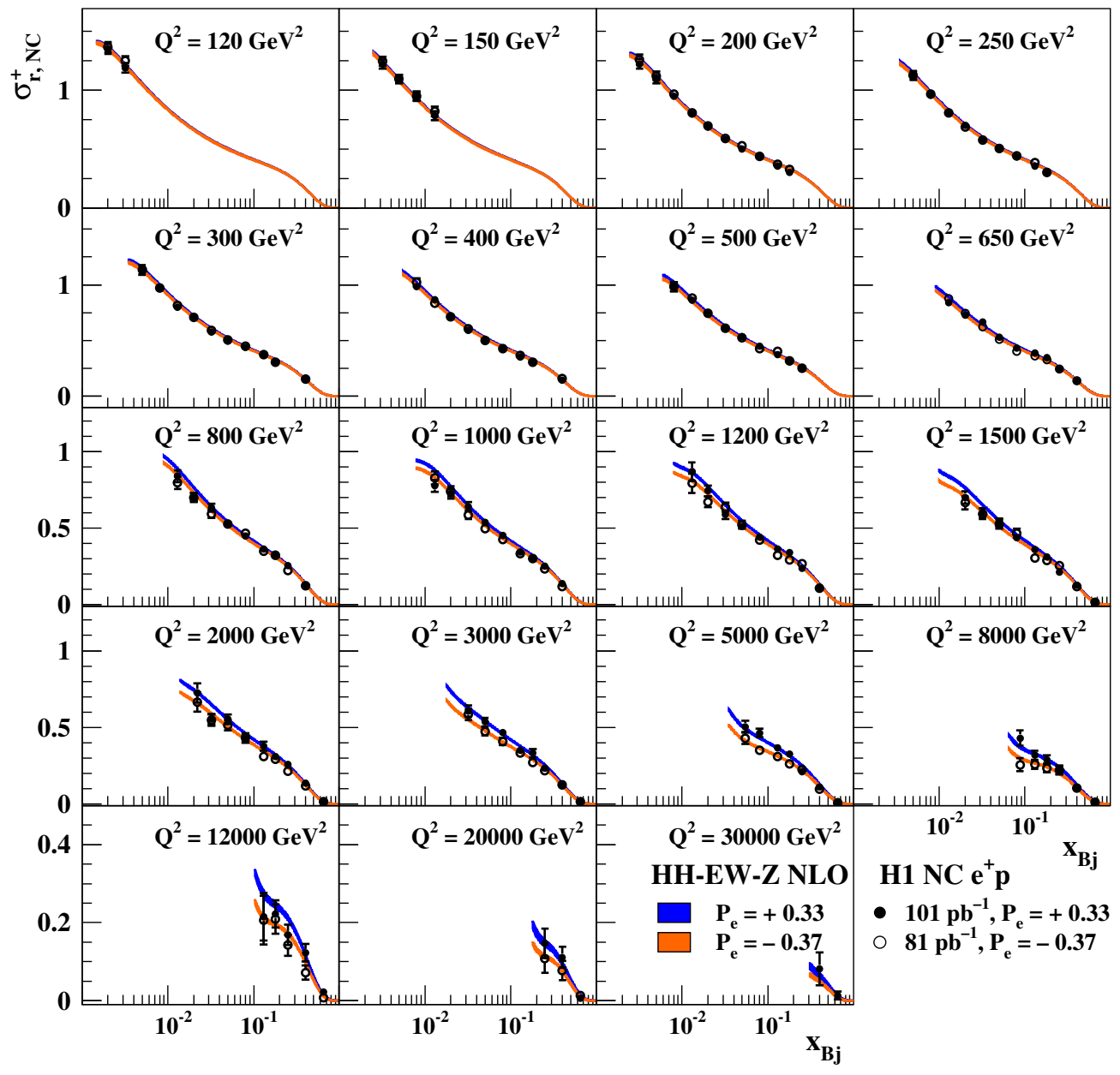

FIG. 2. The NLO predictions of HH-EW-Z compared to the $\mathrm{H} 1 e^{+} p$ NC DIS reduced cross sections $\sigma_{r, \mathrm{NC}}^{+}$for positively and negatively polarized beams plotted as a function of $x_{\mathrm{Bj}}$ at fixed values of $Q^{2}$. The closed (open) circles represent the H1 data for positive (negative) polarization. The bands indicate the full uncertainties on the predictions of HH-EW-Z. 


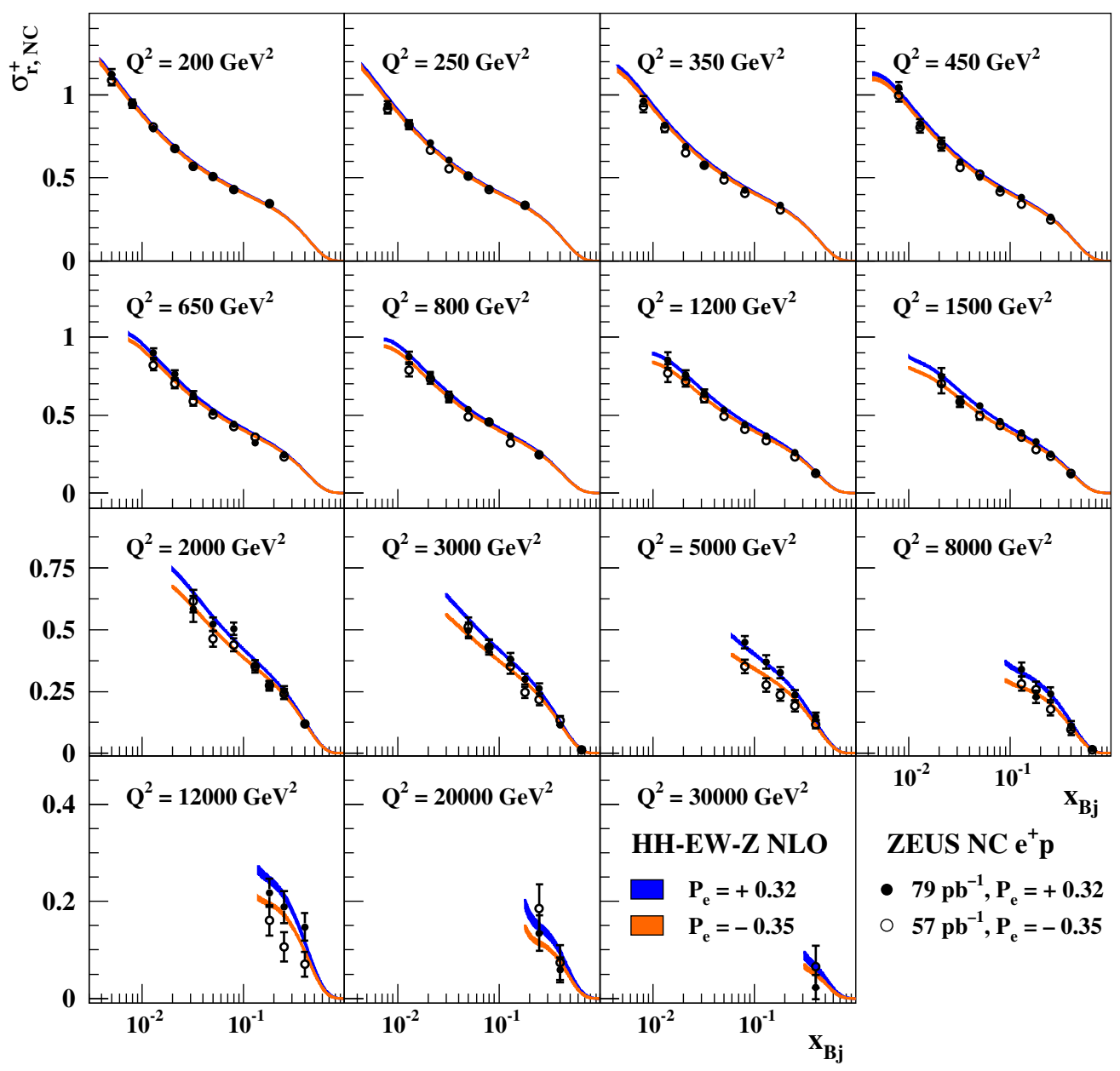

FIG. 3. The NLO predictions of HH-EW-Z compared to the ZEUS $e^{+} p$ NC DIS reduced cross sections, $\sigma_{r, \mathrm{NC}}^{+}$, for positively and negatively polarized beams plotted as a function of $x_{\mathrm{Bj}}$ at fixed values of $Q^{2}$. The closed (open) circles represent the ZEUS data for positive (negative) polarization. The bands indicate the full uncertainties on the predictions of HH-EW-Z.

$$
B_{i}^{0}\left(Q^{2}\right)=-2 e_{i} a_{i} a_{e} \chi_{Z}+4 a_{i} a_{e} v_{i} v_{e} \chi_{Z}^{2} .
$$

The polarized pieces are given by

$$
\begin{gathered}
\tilde{F}_{2}{ }^{P}=\sum_{i} A_{i}^{P}\left(Q^{2}\right)\left[x q_{i}\left(z, Q^{2}\right)+x \bar{q}_{i}\left(x, Q^{2}\right)\right], \\
x \tilde{F}_{3}^{P}=\sum_{i} B_{i}^{P}\left(Q^{2}\right)\left[x q_{i}\left(z, Q^{2}\right)-x \bar{q}_{i}\left(x, Q^{2}\right)\right],
\end{gathered}
$$

where

$$
\begin{aligned}
& A_{i}^{P}\left(Q^{2}\right)=-2 e_{i} v_{i} a_{e} \chi_{Z}+2 v_{e} a_{e}\left(v_{i}^{2}+a_{i}^{2}\right) \chi_{Z}^{2}, \\
& B_{i}^{P}\left(Q^{2}\right)=-2 e_{i} a_{i} v_{e} \chi_{Z}+4 v_{i} a_{i}\left(v_{e}^{2}+a_{e}^{2}\right) \chi_{Z}^{2} .
\end{aligned}
$$

The variable $\chi_{Z}$ denotes the relative strength of $Z$ exchange with respect to photon exchange

$$
\chi_{Z}=\frac{1}{\sin ^{2} 2 \theta_{W}} \frac{Q^{2}}{M_{Z}^{2}+Q^{2}} \frac{1}{1-\Delta R},
$$

where $\Delta R$ accounts for radiative corrections as implemented in EPRC [14]. The structure function $F_{L}$ is only important at high $y$, which is not kinematically accessible for the high- $Q^{2}$ values at which polarization becomes important and thus is not further discussed.

The structure functions $\tilde{F}_{2}$ and $x \tilde{F}_{3}$ described above depend on the vector and axial-vector couplings of the $Z$ boson to the electron $\left(v_{e}, a_{e}\right)$ and to the $u$ - and $d$-type quarks, $v_{u}, a_{u}, v_{d}, a_{d}$. The SM predictions for these couplings are $a_{e}=-1 / 2, \quad v_{e}=-1 / 2+2 \sin ^{2} \theta_{W}$ and $a_{u}=1 / 2, \quad v_{u}=1 / 2-4 / 3 \sin ^{2} \theta_{W}, \quad a_{d}=-1 / 2 \quad$ and $v_{d}=-1 / 2+2 / 3 \sin ^{2} \theta_{W}$. For the HH-EW-Z fit, the couplings $a_{u}, v_{u}, a_{d}, v_{d}$ were free parameters independent of SM predictions.

Given that for the HERA data $\chi_{z} \gg \chi_{z}^{2}$, and that $v_{e} \approx$ 0.04 is very small, it is clear from Eqs. (10), (11), (14), (15), that the terms involving the $a_{i}$ couplings are dominant for 
TABLE II. The results from HH-EW-Z on the axial-vector and vector couplings of the $Z$ boson to $u$ - and $d$-type quarks. Given are the experimental/fit (exp) and total (tot) uncertainties. For comparison, the results of ZEUS-EW-Z are also listed. In addition, results of fits with the PDFs fixed to HH-13p and HERAPDF2.0, for which only the couplings of the $Z$ were free parameters, are given. Also listed are the SM predictions.

\begin{tabular}{|c|c|c|c|c|c|c|c|c|c|c|c|c|}
\hline & $a_{u}$ & Experimental & Total & $a_{d}$ & Experimental & Total & $v_{u}$ & Experimental & Total & $v_{d}$ & Experimental & 1 Total \\
\hline \multirow{2}{*}{ HH-EW-Z } & +0.532 & +0.081 & +0.107 & -0.409 & +0.327 & +0.373 & +0.144 & +0.065 & +0.066 & -0.503 & +0.168 & +0.171 \\
\hline & & -0.058 & -0.063 & & -0.199 & -0.213 & & -0.050 & -0.058 & & -0.093 & -0.103 \\
\hline \multirow[t]{2}{*}{ ZEUS-EW-Z } & +0.50 & +0.09 & +0.12 & -0.56 & +0.34 & +0.41 & +0.14 & +0.08 & +0.09 & -0.41 & +0.24 & +0.25 \\
\hline & \multicolumn{12}{|c|}{ PDF parameters fixed to } \\
\hline \multirow[t]{2}{*}{ HH-13p } & +0.530 & +0.076 & & -0.407 & +0.313 & & +0.145 & +0.063 & & -0.500 & +0.166 & \\
\hline \multirow{2}{*}{\multicolumn{2}{|c|}{ HERAPDF $2.0+0.507$}} & $\begin{array}{l}-0.052 \\
+0.073\end{array}$ & & -0.473 & $\begin{array}{l}-0.193 \\
+0.284\end{array}$ & & +0.155 & $\begin{array}{l}-0.050 \\
+0.062\end{array}$ & & -0.479 & $\begin{array}{l}-0.090 \\
+0.173\end{array}$ & \\
\hline & & -0.047 & & -0.500 & -0.166 & & +0.202 & -0.053 & & -0.351 & -0.110 & \\
\hline
\end{tabular}

the unpolarized data, whereas the $v_{i}$ couplings are dominant for the polarized data. Thus the polarized $\mathrm{H} 1$ data should improve the determination of the couplings $v_{u}$ and $v_{d}$ in comparison to the analysis using only ZEUS polarized data [3].

The data used for the HH-EW-Z fit are the data sets for unpolarized beams originally published by $\mathrm{H} 1$ [21-27] and ZEUS [28-35] and the polarized data sets from $\mathrm{H} 1$ [8] and ZEUS [4-7]. These data are those used as input to the data combination presented by the $\mathrm{H} 1$ and ZEUS collaborations [36]. The data sets with different polarization were added and the resulting cross sections were corrected to zero polarization before they were used as input to the $\mathrm{H} 1$ and ZEUS data combination. A combination of the $\mathrm{H} 1$ and ZEUS polarized data preserving the information on polarization has not been published and therefore this analysis reverts to the original sets of cross sections for polarized beams as published by the individual collaborations, except that the ZEUS cross sections for polarized beams [4-7] are used with updated values of the polarization as published in the ZEUS EW analysis [3].

The reduced cross sections used as input to the analysis were published by the individual collaborations after QED corrections were applied. These corrections are mostly on the percent level, but reach $15 \%$ for a few cross sections. The correction factors were calculated by producing Monte Carlo data sets for which radiative corrections were either turned on or off for comparison. This was done with the program HERACLES [37], interfaced to the hadronization routines within the program DJANGOH [38]. The H1 collaboration published [8] a cross-check with the programs HECTOR [39] and EPRC [14] and concluded that the uncertainties are below $2 \%$ over the complete phase space. The H1 collaboration included these uncertainties in the uncorrelated uncertainties of their published cross sections, whereas the ZEUS collaboration did not assign any uncertainties to their QED corrections in their original
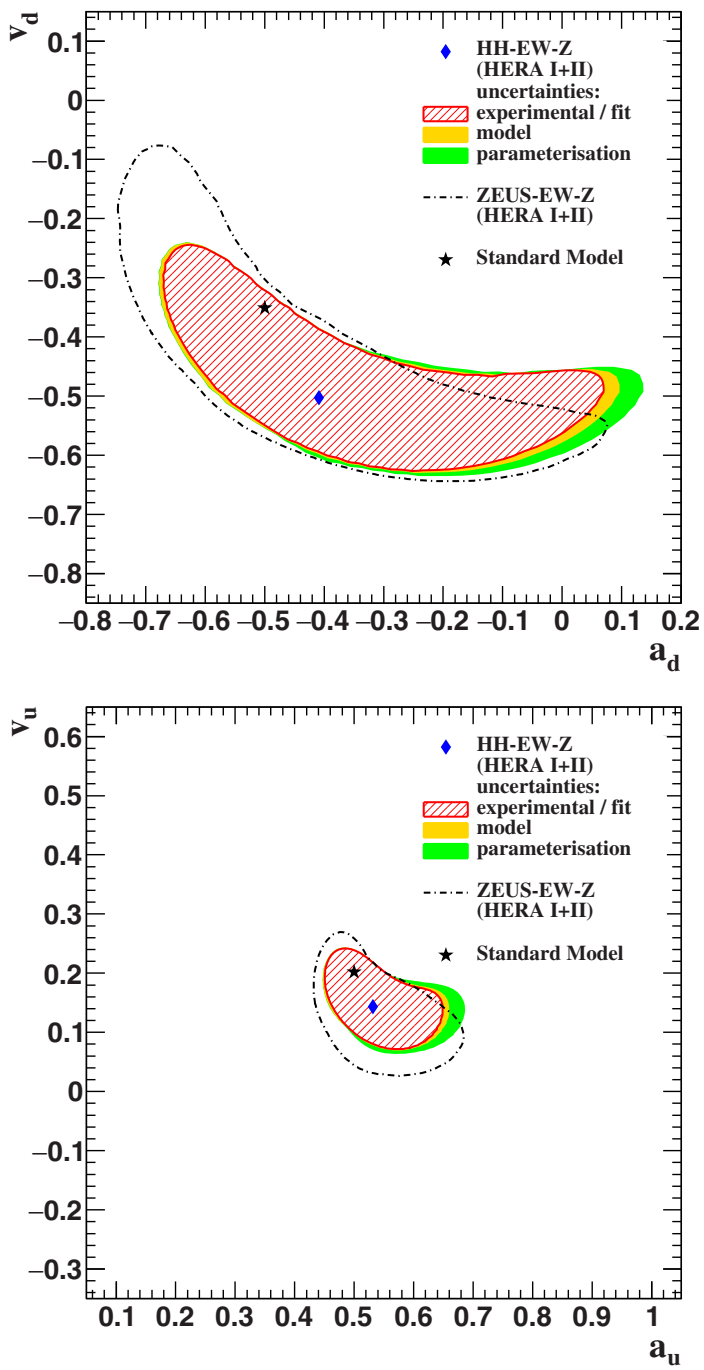

FIG. 4. The $68 \%$ C.L. contours for $a_{d}, v_{d}$ and $a_{u}, v_{u}$ obtained for the HH-EW-Z fit. Also shown are the $68 \%$ C.L. contours for the ZEUS-EW-Z fit with total uncertainties. 
publications. For the present study, an extra uncertainty of the size assigned by $\mathrm{H} 1$ was also added to the uncorrelated uncertainties on the ZEUS cross sections for polarized beams.

\section{THE HH-EW-Z FIT AND THE $\mathrm{Z}$ COUPLINGS}

The PDFs of the HH-EW-Z fit are shown in Fig. 1 with experimental/fit, model and parameterization uncertainties, determined as in the ZEUS-EW-Z fit [3] according to the prescriptions of the HERAPDF2.0 analysis [36]. A 13parameter QCD-only fit with the $Z$ couplings fixed to the $\mathrm{SM}$ values, called $\mathrm{HH}-13 \mathrm{p}$, was performed as a reference; the central values for the PDFs of this fit are also shown in Fig. 1 (top). The PDFs are very similar. The PDF parameters of HH-EW-Z are only weakly correlated to the $Z$ couplings. The full correlation matrix for the $13 \mathrm{PDF}$
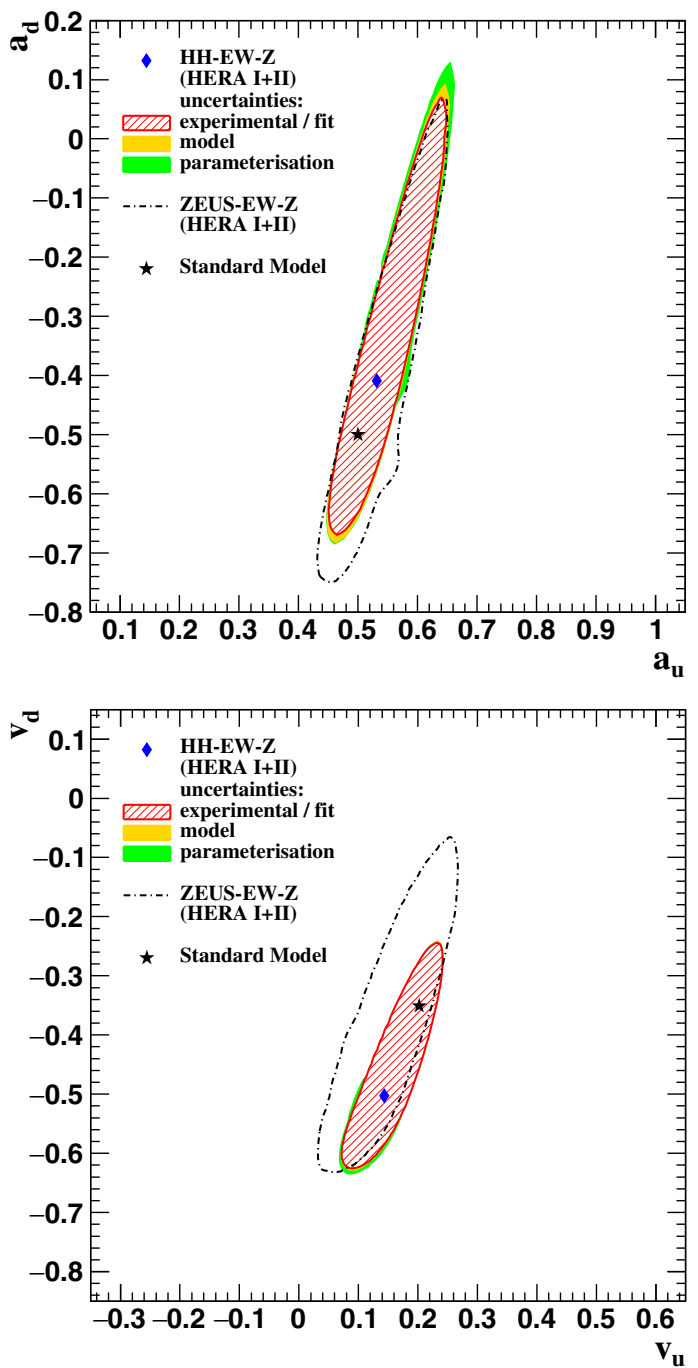

FIG. 5. The $68 \%$ C.L. contours for $a_{u}, a_{d}$ and $v_{u}, v_{d}$ obtained for the HH-EW-Z fit. Also shown are the $68 \%$ C.L. contours for the ZEUS-EW-Z fit with total uncertainties. parameters and the four $Z$ couplings is given in Table I. A comparison of the HH-EW-Z PDFs to the central values of the HERAPDF2.0 NLO is shown in Fig. 1 (bottom). The PDFs are similar within uncertainties. The slight difference in shape of the central values is due to the use of a 14th parameter in the HERAPDF2.0 analysis. The addition of a 14th parameter is included in the parametrization uncertainties quoted for the present analysis.

The $\chi^{2}$ per degree of freedom for HH-EW-Z is $3556 / 3231=1.10$. This can be compared to 1.12 for ZEUS-EW-Z [3] and 1.20 for HERAPDF2.0 NLO [36]. This value of $\chi^{2}$ per degree of freedom is typical for QCD fits to the HERA combined data. The decrease of $\chi^{2}$ when fitting the uncombined data is due to a difference in the effect of the correlations taken into account before and after combination. The description of the data is good.
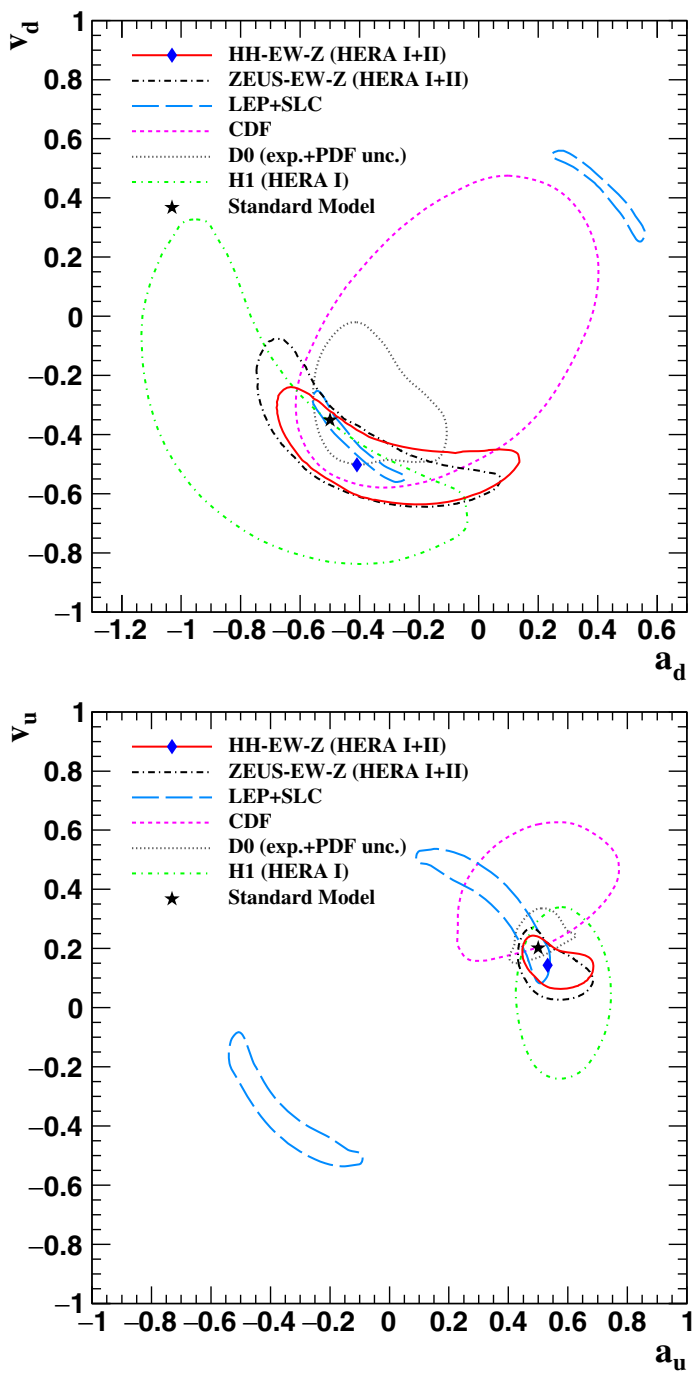

FIG. 6. The $68 \%$ C.L. contours for $a_{d}, v_{d}$ and $a_{u}, v_{u}$ obtained for the HH-EW-Z fit. Also shown are results from ZEUS-EW-Z, HERA I (H1), LEP (ALEPH, OPAL, L3 and DELPHI) plus SLC (SLD) combined, and the Tevatron (CDF and D0). 

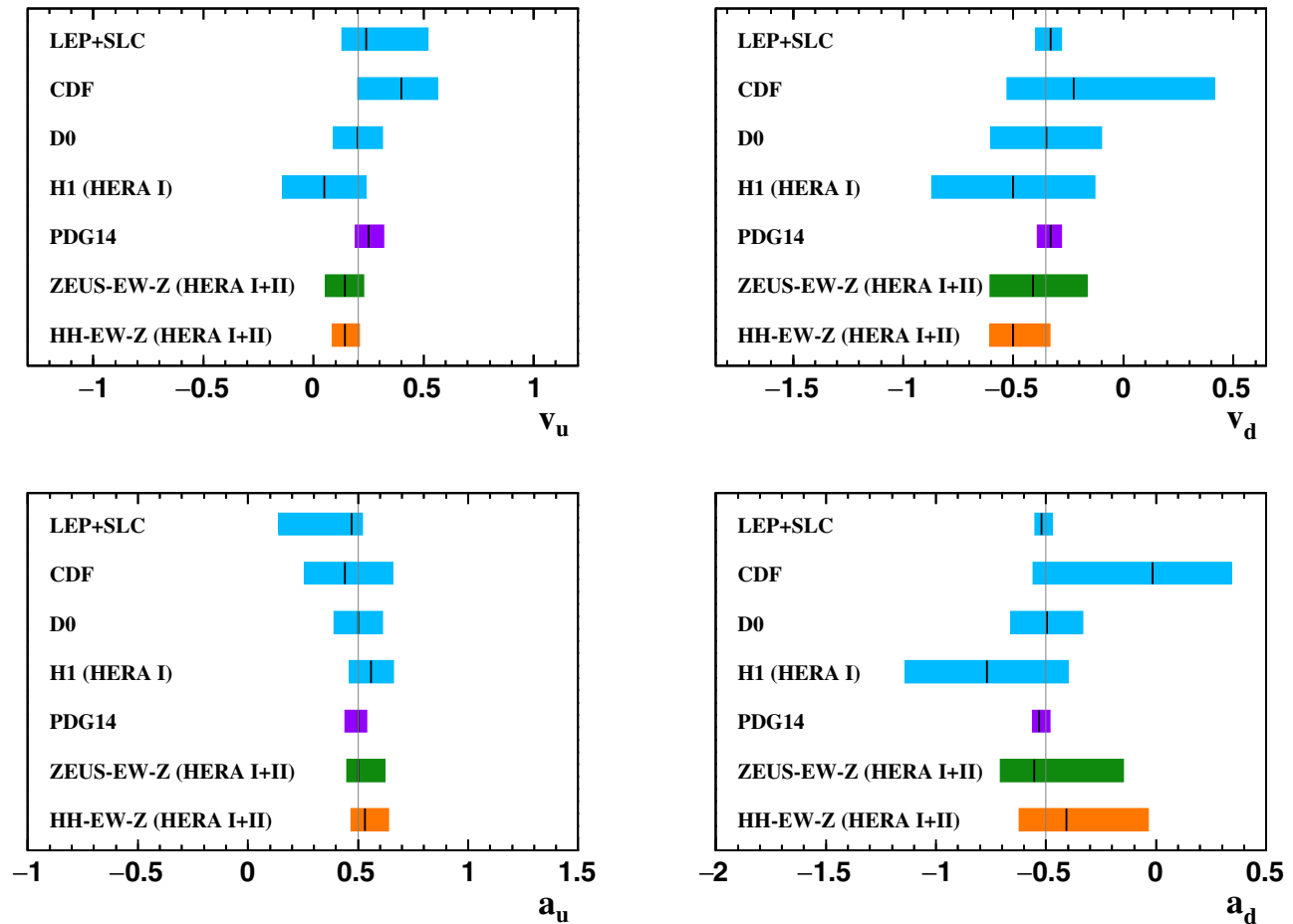

FIG. 7. The values from the HH-EW-Z fit for $a_{d}, a_{u}, v_{d}$ and $v_{u}$ compared to the values from ZEUS-EW-Z and the results from LEP (ALEPH, OPAL, L3 and DELPHI) plus SLC (SLD) combined, the Tevatron (CDF and D0) and HERA I (H1). The PDG14 world average is also shown; this does not contain the measurements from the HH-EW-Z and ZEUS-EW-Z fits based on all HERA data. All results are given with total uncertainties. Vertical black lines in each box indicate central values, the long gray vertical lines indicate the SM predictions.

The predictions of HH-EW-Z are compared to the high-precision $e^{+} p$ NC data from H1 [8] and ZEUS [5] in Figs. 2 and 3 , respectively.

The result of HH-EW-Z for the couplings of the $Z$ boson to $u$ - and $d$-type quarks are

$$
\begin{aligned}
& a_{u}=+0.532_{-0.058}^{+0.081}(\text { experimental } / \mathrm{fit})_{-0.022}^{+0.036}(\text { model })_{-0.008}^{+0.060}(\text { parametrization }), \\
& a_{d}=-0.409_{-0.199}^{+0.327}(\text { experimental } / \mathrm{fit})_{-0.071}^{+0.112}(\text { model })_{-0.026}^{+0.140}(\text { parametrization }), \\
& v_{u}=+0.144_{-0.050}^{+0.065}(\text { experimental } / \mathrm{fit})_{-0.014}^{+0.013}(\text { model })_{-0.025}^{+0.002}(\text { parametrization }), \\
& v_{d}=-0.503_{-0.093}^{+0.168}(\text { experimental } / \mathrm{fit})_{-0.028}^{+0.031}(\text { model })_{-0.036}^{+0.006}(\text { parametrization }) .
\end{aligned}
$$

These values are compared to the results from ZEUS-EW-Z [3] in Table II. They agree within uncertainties. Also listed are SM predictions and values obtained from fits which were performed as cross-checks

(i) a fit in which only the axial-vector and vector couplings of the $Z$ were allowed to vary and the PDF parameters are fixed to those of the 13-parameter QCD-only fit, HH-13p;

(ii) a fit in which only the axial-vector and vector couplings of the $Z$ were allowed to vary and the PDF parameters are fixed to those of HERAPDF2.0 NLO.

Only experimental/fit uncertainties were considered for these cross-checks. The values agree within the experimental uncertainties with the result from HH-EW-Z.
Profile likelihood contours at $68 \%$ C.L. for the couplings were obtained as described in the ZEUS publication [3]. They are shown ${ }^{4}$ for $a_{u}, v_{u}$ and $a_{d}, v_{d}$ in Fig. 4 and for $a_{u}$, $a_{d}$ and $v_{u}, v_{d}$ in Fig. 5. These figures demonstrate very clearly that the HERA data constrain the couplings of the $Z$ boson to the $u$ quark significantly better than the couplings to the $d$ quark. This is due to the larger $u$ valence content of the proton and the larger charge of the $u$ quark. The couplings as determined by HH-EW-Z are compatible with the SM. Figure 6 shows the $68 \%$ C.L. contours from HHEW-Z, together with the contours from ZEUS-EW-Z [3]

\footnotetext{
${ }^{4}$ Numerical information is available as additional material for this publication.
} 
and the measurements from LEP + SLC [40], the Tevatron [41,42] and HERA I (H1) [43]. The fits HH-EW-Z and ZEUS-EW-Z are based both on HERA I and HERA II data and were not included in the combinations for PDG14 [18]. The PDG values and all measurements are compared in Fig. 7. The HH-EW-Z results on the axial-vector and vector couplings to $u$-type quarks are the most precise results published from a single process. As expected, the vector couplings from $\mathrm{HH}-\mathrm{EW}-\mathrm{Z}$ are significantly more accurate than from ZEUS-EW-Z.

The ZEUS collaboration also presented [3] measurements of the electroweak mixing angle and $M_{W}$. These EW parameters were determined using both $\mathrm{NC}$ and $\mathrm{CC}$ cross sections. The factor $\chi_{Z}$, which depends on $\sin ^{2} \theta_{W}$, affects the contributions to the NC cross sections for both polarized and unpolarized beams. The dependence of the CC cross sections on $\sin ^{2} \theta_{W}$ and $M_{W}$ is described through the factor $\frac{G_{F}^{2} M_{W}^{4}}{2 \pi x_{\mathrm{Bj}}\left(Q^{2}+M_{W}^{2}\right)^{2}}$, where $G_{F}=$ $\frac{\pi \alpha}{\sqrt{2} \sin ^{2} \theta_{W} M_{W}^{2}} \frac{1}{1-\Delta R}$ and polarization enters through the multiplicative factors $\left(1 \pm P_{e}\right)$ for $e^{ \pm}$scattering, respectively. Thus, the information contained in the CC cross sections is also not uniquely dependent on polarization. Therefore, fits including the $\mathrm{H} 1$ polarized data produce no significant improvement in the accuracy of the mixing angle and $M_{W}$ compared to the ZEUS-EW analysis [3].

Two fits were performed as cross-checks with the 13 PDF parameters fixed to those of $\mathrm{HH}-13 \mathrm{p}$ and either $\sin ^{2} \theta_{W}$ or $M_{W}$ as free parameters. The results are compatible with those of the ZEUS EW fits within experimental/fit uncertainties

$$
\begin{aligned}
\sin ^{2} \theta_{W} & =0.2255 \pm 0.0011(\text { experimental/fit }) \quad \mathrm{HH} \text { EW } \\
\sin ^{2} \theta_{W} & =0.2252 \pm 0.0011(\text { experimental/fit }) \quad \mathrm{ZEUS} \mathrm{EW}, \\
M_{W} & =(80.74 \pm 0.28(\text { experimental/fit })) \mathrm{GeV} \text { HH EW } \\
M_{W} & =(80.68 \pm 0.28(\text { experimental/fit })) \mathrm{GeV} \quad \text { ZEUS EW. }
\end{aligned}
$$

A simultaneous fit to the 13 PDF parameters and both $\sin ^{2} \theta_{W}$ and $M_{W}$ also yielded results compatible with the results presented by ZEUS [3]. Since the sensitivity with respect to the ZEUS EW fits was not significantly improved, the detailed studies on $\sin ^{2} \theta_{W}$ and $M_{W}$ presented in the ZEUS paper were not repeated.

\section{SUMMARY AND CONCLUSIONS}

The results of a combined electroweak and QCD fit to all available HERA inclusive DIS cross sections, taking into account beam polarization for both the $\mathrm{H} 1$ and ZEUS data, have been presented. The results on the couplings of the $Z$ boson to $u$ - and $d$-type quarks are

$$
\begin{aligned}
& a_{u}=+0.532_{-0.058}^{+0.081}(\text { experimental } / \mathrm{fit})_{-0.022}^{+0.036}(\text { model })_{-0.008}^{+0.060}(\text { parametrization }), \\
& a_{d}=-0.409_{-0.199}^{+0.327}(\text { experimental } / \mathrm{fit})_{-0.071}^{+0.112}(\text { model })_{-0.026}^{+0.140}(\text { parametrization }), \\
& v_{u}=+0.144_{-0.050}^{+0.065}(\text { experimental } / \mathrm{fit})_{-0.014}^{+0.013}(\text { model })_{-0.025}^{+0.002}(\text { parametrization }), \\
& v_{d}=-0.503_{-0.093}^{+0.168}(\text { experimental } / \mathrm{fit})_{-0.028}^{+0.031}(\text { model })_{-0.036}^{+0.006}(\text { parametrization }) .
\end{aligned}
$$

These results are compatible with the Standard Model. The exploitation of all available data for polarized beams provides very accurate determinations of the $Z$-boson couplings. The couplings to the $u$-type quarks are the most precise values published for a single process.

\section{ACKNOWLEDGMENTS}

We are grateful to our ZEUS and H1 colleagues who supported this work. We especially thank Achim Geiser for the many fruitful discussions. We thank our funding agencies, the Alexander von Humboldt-Stiftung and the Max-PlanckSociety, for financial support and DESY for the hospitality extended to the non-DESY authors. 
[1] A. M. Cooper-Sarkar and R. Devenish, Deep Inelastic Scattering (Oxford University Press, Oxford, 2011)), ISBN 978-0-19-960225-4.

[2] H. Abramowicz and A. Caldwell, Rev. Mod. Phys. 71, 1275 (1999).

[3] H. Abramovicz et al. (ZEUS Collaboration) Phys. Rev. D 93, 092002 (2016).

[4] S. Chekanov et al. (ZEUS Collaboration), Eur. Phys. J. C 62, 625 (2009).

[5] H. Abramowicz et al. (ZEUS Collaboration), Phys. Rev. D 87, 052014 (2013).

[6] S. Chekanov et al. (ZEUS Collaboration), Eur. Phys. J. C 61, 223 (2009).

[7] H. Abramowicz et al. (ZEUS Collaboration), Eur. Phys. J. C 70, 945 (2010).

[8] F. Aaron et al. (H1 Collaboration), J. High Energy Phys. 09 (2012) 061.

[9] V. Gribov and L. Lipatov, Sov. J. Nucl. Phys. 15, 438 (1972).

[10] V. Gribov and L. Lipatov, Sov. J. Nucl. Phys. 15, 675 (1972).

[11] L. Lipatov, Sov. J. Nucl. Phys. 20, 94 (1975).

[12] Y. Dokshitzer, Sov. Phys. JETP 46, 641 (1977).

[13] G. Altarelli and G. Parisi, Nucl. Phys. B126, 298 (1977).

[14] H. Spiesberger, in Proceedings Workshop on Future Physics at HERA, edited by G. Ingelman, A. De Roeck, and R. Klanner (DESY, Hamburg, Germany, 1995).

[15] R. S. Thorne and R. G. Roberts, Phys. Rev. D 57, 6871 (1998).

[16] R. S. Thorne, Phys. Rev. D 73, 054019 (2006).

[17] R. S. Thorne, Phys. Rev. D 86, 074017 (2012).

[18] K. A. Olive et al. (Particle Data Group), Chin. Phys. C 38, 090001 (2014).

[19] A. D. Martin, W. J. Stirling, R. S. Thorne, and G. Watt, Eur. Phys. J. C 63, 189 (2009).

[20] S. Alekhin et al. Eur. Phys. J. C 75, 304 (2015).

[21] V. Andreev et al. (H1 Collaboration), Eur. Phys. J. C 74, 2814 (2014).

[22] F. Aaron et al. (H1 Collaboration), Eur. Phys. J. C 71, 1579 (2011).

[23] C. Adloff et al. (H1 Collaboration), Eur. Phys. J. C 13, 609 (2000).

[24] C. Adloff et al. (H1 Collaboration), Eur. Phys. J. C 19, 269 (2001).
[25] C. Adloff et al. (H1 Collaboration), Eur. Phys. J. C 30, 1 (2003).

[26] F. Aaron et al. (H1 Collaboration), Eur. Phys. J. C 64, 561 (2009).

[27] F. Aaron et al. (H1 Collaboration), Eur. Phys. J. C 63, 625 (2009).

[28] H. Abramowicz et al. (ZEUS Collaboration), Phys. Rev. D 90, 072002 (2014).

[29] J. Breitweg et al. (ZEUS Collaboration), Eur. Phys. J. C 7, 609 (1999).

[30] J. Breitweg et al. (ZEUS Collaboration), Eur. Phys. J. C 12, 411 (2000); 27, 305(E) (2003).

[31] S. Chekanov et al. (ZEUS Collaboration), Eur. Phys. J. C 21, 443 (2001).

[32] S. Chekanov et al. (ZEUS Collaboration), Phys. Lett. B 539, 197 (2002); 552, 308(E) (2003).

[33] S. Chekanov et al. (ZEUS Collaboration), Eur. Phys. J. C 28, 175 (2003).

[34] S. Chekanov et al. (ZEUS Collaboration), Eur. Phys. J. C 32, 1 (2003).

[35] S. Chekanov et al. (ZEUS Collaboration), Phys. Rev. D 70, 052001 (2004).

[36] H. Abramovicz et al. (ZEUS and H1 Collaborations), Eur. Phys. J. C 75, 580 (2015).

[37] A. Kwiatkowski, H. Spiesberger, and H.-J. Möhring, Comput. Phys. Commun. 69, 155 (1992).

[38] G. A. Schuler and H. Spiesberger, Proceedings Workshop on Physics at HERA, edited by W. Buchmüller and G. Ingelman, Vol. 3, p. 1419 (DESY, Hamburg, Germany, 1991).

[39] A. Arbuzov, D. Bardin, J. Blümlein, L. Kalinovskaya, and T. Riemann, Comput. Phys. Commun. 94, 128 (1996).

[40] G. Abbiendi et al. (ALEPH, DELPHI, L3 and OPAL Collaborations, SLD Collaboration, (LEP Electroweak Working Group, SLD Electroweak, and Heavy Flavor Groups)), Phys. Rep. 427, 257 (2006).

[41] V. Abazov et al. (D0 Collaboration), Phys. Rev. D 84, 012007 (2011).

[42] D. Acosta et al. (CDF Collaboration), Phys. Rev. D 71, 052992 (2005).

[43] A. Aktas et al. (H1 Collaboration), Phys. Lett. B 632, 35 (2006). 